\title{
Properties of High Lying Levels in ${ }^{199} \mathrm{Au}$
}

\author{
E. BAShandy and G. M. EL-SAYAD \\ Nuclear Physics Department, Atomic Energy Establishment, Cairo, U.A.R.
}

(Z. Naturforschg. 22 a, 1538-1542 [1967] ; received 15 March 1967)

\begin{abstract}
The energy level spectrum of ${ }^{199} \mathrm{Au}$ excited in the decay of the $30 \mathrm{~min}$ activity of ${ }^{199} \mathrm{Pt}$ was investigated by means of a high resolution, double focussing $\beta$-ray spectrometer. The $\mathrm{K}$ - and $\mathrm{L}$ conversion ratios of the 196 and $221 \mathrm{keV} \gamma$ rays show that both are pure E2 transitions. The internal K-conversion coefficients of the 541,790 and $960 \mathrm{keV} \gamma$ rays were determined by the internalexternal conversion method, and the following results were obtained:

$$
a_{\mathrm{K}}(541)=0.059 \pm 0.007 ; \quad a_{\mathrm{K}}(790)=0.023 \pm 0.003 ; \quad a_{\mathrm{K}}(960)=0.062 \pm 0.008 .
$$

The multipolarity assignments of these transitions were studied on the basis of the conversion coefficients. These data and the studies of other workers were combined to obtain the spins and parities $5 / 2+, 1 / 2+, 3 / 2+$ and $9 / 2+$ for the $541,737,790$ and $960 \mathrm{keV}$ levels in ${ }^{199} \mathrm{Au}$.
\end{abstract}

Recently Joshi et al. ${ }^{\mathbf{1}}$ have investigated the levels in ${ }^{199} \mathrm{Au}$ with particular emphasis on the isomeric state which was found to be at $475 \mathrm{keV}$. In all of the previous studies a scintillation either single or coincidence spectrum was determined and used in the elucidation of the decay scheme of ${ }^{199} \mathrm{Pt}$. The spins and parities of most of the levels in ${ }^{199} \mathrm{Au}$ were assigned from the study of $\log f t$ values of the $\beta$ branches populating them, as well as from the analogy of the levels in odd-mass gold nuclei. For several levels of ${ }^{199} \mathrm{Au}$ the spin and parity assignments are uncertain. The low-lying level schemes of ${ }^{193} \mathrm{Au},{ }^{195} \mathrm{Au}$ and ${ }^{197} \mathrm{Au}$ are found to be similar, but that of ${ }^{199} \mathrm{Au}$, according to previous workers, differs considerably. However, after the extensive studies of coincidence measurements done by JosHi et al. ${ }^{1}$ a comparable decay scheme was proposed. They concluded that $75 \mathrm{keV}$ is the energy of the lowest excited state in ${ }^{199} \mathrm{Au}$, and not $197 \mathrm{keV}$ as was reported ${ }^{2}$ before.

Since the neutrons are being filled pairwise into the next major shell $(N=126)$ the addition of two neutrons to ${ }^{197} \mathrm{Au}$ should not have a very large effect on the motion of the $79^{\text {th }}$ proton in the lower major shell $(p=82)$. Therefore one should expect a lowlying level scheme for ${ }^{199} \mathrm{Au}$ similar to that of the other odd-mass gold nuclei, as suggested by JosHI et al. ${ }^{1}$.

The level schemes of odd-mass gold nuclei have been a subject of theoretical interest. KIssLINGER and SoRENSEN ${ }^{3}$ have calculated them taking into account

1 M. C. Joshi, B. V. Thosar, and K. G. Prasad, Nucl. Phys. 52, 301 [1964].

2 J. M. Le-Blanc, J. M. Cork, and S. B. Burson, Phys. Rev. 104, 1670 [1956]. the residual nuclear interactions. From the level systematics in the mass region $190<A<202$ it is noted that even nuclei of platinum exhibit a vibrational level spectrum at least up to two phonon states with surface vibrational energy ${ }^{1} h \cdot \omega=350 \mathrm{keV}$. This suggests that the level scheme of odd-mass gold nuclei can also be described as due to the coupling of the odd particle motion with the surface vibrations of the even core.

In view of such interest, it was decided to examine experimentally the excited states of ${ }^{199} \mathrm{Au}$ and to compare them with the theoretical predictions. The present study is an attempt to assign spins and character to states not yet fully identified.

In this paper we report measurements of several internal conversion coefficients from which information on the states at $541,737,790$ and $960 \mathrm{keV}$ can be deduced. The internal-external conversion method was used. This method is very suitable for complex decays such as that of ${ }^{199} \mathrm{Pt}$.

\section{Experimental Procedures}

The excited levels in ${ }^{199} \mathrm{Au}$ were studied from the decay of the $30 \mathrm{~min}{ }^{199} \mathrm{Pt}$. Energies and relative intensities of the internal and external conversion lines emitted in the decay were measured by means of a high resolution iron-free, double-focussing, $\beta$-ray spectrometer ${ }^{4}\left(\varrho_{0}=50 \mathrm{~cm}\right)$. With this instrument relative momentum measurements could be

${ }^{3}$ L. S. Kisslinger and R. A. Sorensen, Rev. Mod. Phys. 35, 853 [1963].

4 M. S. EL-NESR and G. M. EL-SAYAD, Int. Rep. 2 UAR, A.E.E. 1965. 
made with an accuracy of a few parts in $10^{5}$. With a $0.2 \times 2 \mathrm{~cm}^{2}$ source and a $2 \mathrm{~mm}$ detector slit a resolution of $\sim 0.15 \%$ is obtained. The detector employed in the present studies was a G. M. counter with a $2.1 \mathrm{mg} / \mathrm{cm}^{2}$ mica end window.

\section{Source Preparation}

The platinum activity was produced by thermal neutron bombardement of natural platinum metal over a period of 5 hours in the UAR Reactor at Inchass. The flux was about $10^{13}$ neutrons $/ \mathrm{cm}^{2} \mathrm{~s}$. For the internal-conversion studies, platinum was uniformaly sputtered on aluminium foil of thickness $\sim 1 \mathrm{mg} / \mathrm{cm}^{2}$. The sputtered material was distributed in a rectangular area $\left(0.2 \times 2 \mathrm{~cm}^{2}\right)$. The thickness of the material deposited was estimated to be $\approx 100 \mu \mathrm{g} / \mathrm{cm}^{2}$.

For the external-conversion measurements rectangular sources $2 \mathrm{~mm}$ wide, $1.6 \mathrm{~cm}$ long, and $0.1 \mathrm{~mm}$ thick were used. During the measurements the source was embedded between $\beta$ absorbing copper sheets with a thickness of $1 \mathrm{~mm}$. The resulting "Sandwich" was then mounted closely behind a uranium converter. The uranium converter $(5 \times 30$ $\mathrm{mm}^{2}$ ) was of thickness $2.9 \mathrm{mg} / \mathrm{cm}^{2}$.

\section{Measurements}

The internal conversion spectra for the 196, 221, 541,790 and $960 \mathrm{keV}$ transitions were studied carefully in the double focussing, $\beta$-ray spectrometer. As we used natural platinum targets, conversion lines from several isotopes were to be expected. In point of fact, the purity of mass separated sources did not compensate for the great loss of material in the mass separator and it was generally found preferable to use unseparated sources and to work with better resolution in the spectrometer. Since unseparated sources were generally used the isotopic identification of the observed lines constituted a difficult and unavoidable problem. However, the conversion spectra of several isotopes are known and their conversion lines could be estimated. Also the shortening in the duration of irradiation lessens the contributions of long lived platinum activities, mainly ${ }^{197} \mathrm{Pt}$ $(18 \mathrm{~h}),{ }^{195 \mathrm{~m}} \mathrm{Pt}(3.5 \mathrm{~d})$ and ${ }^{193 \mathrm{~m} P t}(4.3 \mathrm{~d})$. The Linternal conversion lines of the $196 \mathrm{keV}$ transition were measured carefully, see Fig. 1 . The K-conversion line could not be determined absolutely since it is superimposed on other lines. Also the internal

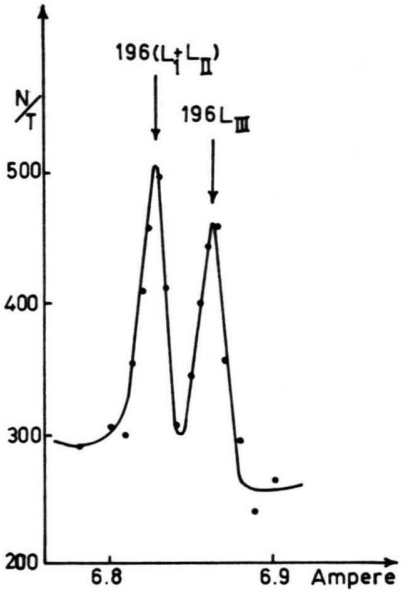

Fig. 1. The L-conversion lines of the $196 \mathrm{keV}$ transition in ${ }^{199} \mathrm{Au}$.

conversion spectrum including the K-conversion lines of the transitions 221, 541, 790 and $960 \mathrm{keV}$ as well as the L-conversion lines of $221 \mathrm{keV}$ transition were recorded. Because of the small amount of ${ }^{199} \mathrm{Pt}$ produced in each irradiation and its fairly short half-life $(30 \mathrm{~min})$, only a small interval of the spectrum could be recorded using one source. Each conversion line was measured at least twice. The data were corrected for half-life. Energy calibrations were performed using ${ }^{170} \mathrm{Tm},{ }^{198} \mathrm{Au}$ and ${ }^{137} \mathrm{Cs}$ as standard sources. The intensities of the electron lines were determined by integrating the areas of peaks on an $N / P$ versus $P$ plot $(P$ is the momentum). The errors in the intensities arose mainly from uncertainties in resolving neighbouring lines. In the lowest energy region, however, larger errors were introduced by the difficulty in correcting for window absorption and by electron straggling at the source. The standard error of these intensities is believed to be $\approx 4 \%$ for the intense lines and more than $10 \%$ for the weaker lines.

The external conversion spectra were measured in order to determine the relative $\gamma$ intensity for the transitions in question. The emission of photoelectrons from the converter is not isotropic and in order to reduce scattering effects the external conversion process must then take place in very thin layers of material. As is well known, the photon intensity from a converter of uniform density is proportional to the total cross section $\tau_{\mathrm{x}}$ (for the particular atomic shell and transition energy) and a factor $f$, which depends on the character of the appropriate photoelectric angular distribution and on the 
details of the experimental arrangement of the $\gamma$-ray source and the photo-electric converter inside the spectrometer. According to Hultberg ${ }^{5}$ the intensity of a $\gamma$ ray can then be expressed as:

$$
I_{\gamma}=C \cdot A_{\gamma} / f \tau
$$

where $C$ is a constant depending on the converter thickness, source strength and the instrumental transmission factor and $A_{\gamma}$ is the measured intensity of a photoline taken as the area under the photoconversion peak after normalizing to unit momentum interval. The relative $\gamma$ intensities for the 221, 541, 790 and $960 \mathrm{keV}$ transitions have been determined and compared with the previous results obtained by scintillation technique, see Table 1. An example of the external conversion lines is shown in Fig. 2.

\begin{tabular}{|c|c|c|c|c|}
\hline $\begin{array}{c}\gamma \text { energy } \\
(\mathrm{keV})\end{array}$ & $\begin{array}{l}\text { Prasad } \\
\text { from } \\
\text { Ge(Li) }\end{array}$ & $\begin{array}{l}\text { al. }{ }^{6} \\
\text { from } \\
\mathrm{NaI}(\mathrm{Tl})\end{array}$ & BlaCK ${ }^{7}$ & $\begin{array}{c}\text { External } \\
\text { conversion } \\
\text { present wo rk }\end{array}$ \\
\hline 221 & $2.5 \pm 0.5$ & & 2.2 & $3.2 \pm 0.5$ \\
\hline 541 & 100 & 100 & 100 & 100 \\
\hline 790 & $8 \pm 1$ & 10 & 7.6 & $8.3 \pm 1.1$ \\
\hline 960 & $6 \pm 1$ & 8 & 6.3 & $6.1 \pm 1.0$ \\
\hline
\end{tabular}

Table 1. Relative intensities of some $\gamma$ rays in ${ }^{199} \mathrm{Au}$.

By means of the photon intensities and the conversion electron data of the 221, 541, 790 and $960 \mathrm{keV}$ transitions, the absolute conversion coefficients have been calculated and are presented in Table 2. Normalization between the two series of data is obtained by assuming that the $221 \mathrm{keV} \gamma$ ray is a pure $\mathrm{E} 2$ transition as is indicated by the $\mathrm{K} / \mathrm{L}$ and L-subshell ratios, see Fig. 3. The basic quantity for the determination of the internal conversion coef-

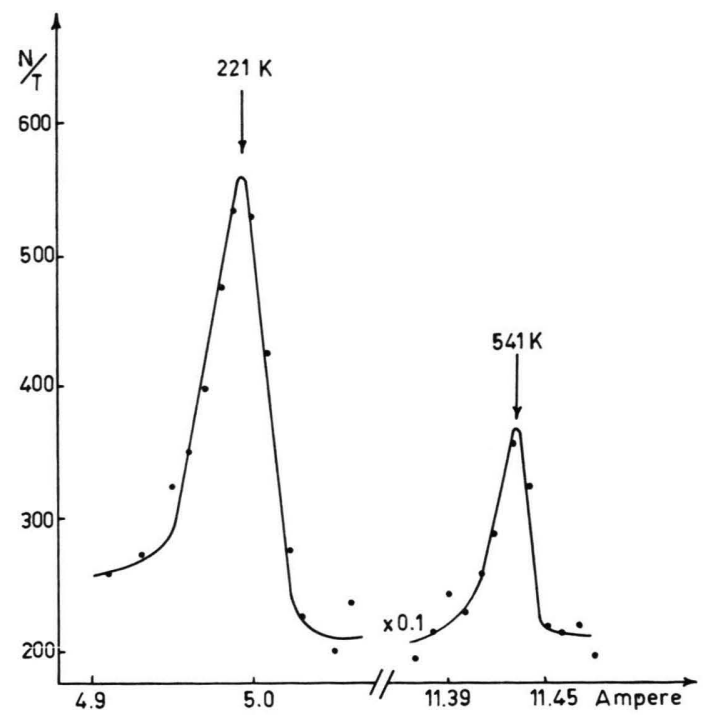

Fig. 2. The external K-conversion lines of the 221 and $541 \mathrm{keV}$ transitions.

ficient is the integrated photoelectric cross-section $\tau$. It is therefore essential to have access to accurate tables of $\tau$. At present the most accurate calculations are those for $\tau_{\mathrm{K}}$ by Hultberg et al. ${ }^{8}$. They are corrected to any order in $\alpha_{z}$ but neglect the effect of screening. The correction for the latter effect is rather small for the $\mathrm{K}$ shell and can partially be corrected for.

A rectangular source and converter is the most useful geometry for measurements with a magnetic spectrometer of a double-focussing type. Correction factors $f_{\mathrm{k}}$ including all the effects have been computed with the electronic computer BESK, Stockholm, for the energies mentioned and considering the specific geometry used in the present experiment.

\begin{tabular}{|c|c|c|c|c|c|c|c|c|c|}
\hline \multirow{2}{*}{$\gamma \underset{(\mathrm{keV})}{\gamma \text { energy }}$} & \multirow{2}{*}{$\begin{array}{l}\text { Conversion } \\
\text { lines }\end{array}$} & \multicolumn{5}{|c|}{ Theoretical values 9} & \multirow[b]{2}{*}{ M 3} & Experimental & \multirow{2}{*}{$\begin{array}{l}\text { Multi- } \\
\text { polarity }\end{array}$} \\
\hline & & E 1 & E 2 & E 3 & M 1 & M 2 & & values & \\
\hline \multirow[t]{2}{*}{196} & $\left(\mathrm{~L}_{\mathrm{I}}+\mathrm{L}_{\mathrm{II}}\right) / \mathrm{L}_{\mathrm{III}}$ & 5.84 & 1.93 & 2.07 & 123.66 & 9.33 & 2.05 & $1.76 \pm 0.11$ & \multirow[t]{2}{*}{ E 2} \\
\hline & $\mathrm{K} / \mathrm{L}$ & 5.83 & 1.26 & 0.28 & 5.85 & 3.75 & 1.79 & $1.18 \pm 0.12$ & \\
\hline \multirow[t]{2}{*}{221} & $\mathrm{~K} /\left(\mathrm{L}_{\mathrm{I}}+\mathrm{L}_{\mathrm{II}}\right)$ & 67.49 & 1.82 & 0.40 & 5.90 & 4.10 & 2.55 & $1.70 \pm 0.18$ & \multirow[t]{2}{*}{ E 2} \\
\hline & $\left(\mathrm{L}_{\mathrm{I}}+\mathrm{L}_{\mathrm{II}}\right) / \mathrm{L}_{\mathrm{III}}$ & 6.38 & 2.26 & 2.28 & 125.13 & 10.68 & 2.39 & $2.08 \pm 0.21$ & \\
\hline 541 & $\alpha_{\mathrm{K}}$ & 0.006 & 0.016 & 0.039 & 0.057 & 0.160 & 0.368 & $0.059 \pm 0.007$ & M 1 \\
\hline 790 & $\alpha_{K}$ & 0.0029 & 0.007 & 0.016 & 0.021 & 0.054 & 0.108 & $0.023 \pm 0.003$ & M 1 \\
\hline 960 & $\alpha_{K}$ & 0.002 & 0.005 & 0.011 & 0.013 & 0,031 & 0.060 & $0.062 \pm 0.008$ & M 3 \\
\hline
\end{tabular}

Table 2. Internal conversion coefficients and multipolarity of some transitions in ${ }^{199} \mathrm{Au}$. 5 S. Hultberg, Arkiv Fysik 15, 307 [1959].
${ }^{6}$ K. G. Prasad, R. P. Sharma, and B. V. Thosar, Phys. Rev.
149, 980 [1966].
7 W. W. Black, Bull. Am. Phys. Soc. 10, 481 [1965].

8 S. Hultberg, B. NAgel, and P. Olssen, Arkiv Fysik 21, 555 [1961]. 


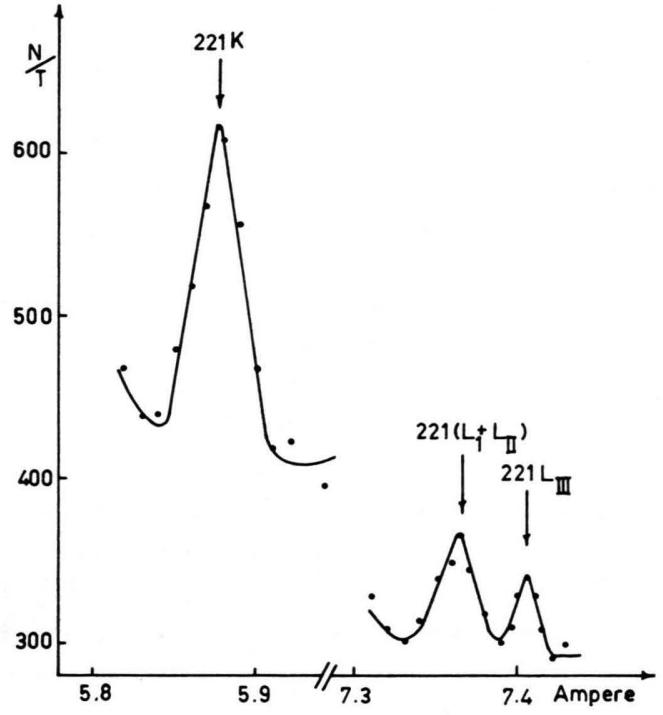

Fig. 3. The internal conversion lines of the $221 \mathrm{keV}$ transition in ${ }^{199} \mathrm{Au}$ (not decay corrected).

\section{Results and Discussion}

As the main purpose of this investigation is the determination of the properties of highly excited levels in ${ }^{199} \mathrm{Au}$, we concentrated the study on the measurement of internal and external conversion spectra of the 196, 221, 541, 790 and $960 \mathrm{keV}$ transitions. Owing to the lack of a high-precision internal conversion electron singlet spectrum, the multipolarity assignments could not be made unambiguously from $\mathrm{K} / \mathrm{L}$ and $\mathrm{L}_{1}+\mathrm{L}_{11} / \mathrm{L}_{111}$ ratios except in the case of the 196 and $221 \mathrm{keV}$ transi-

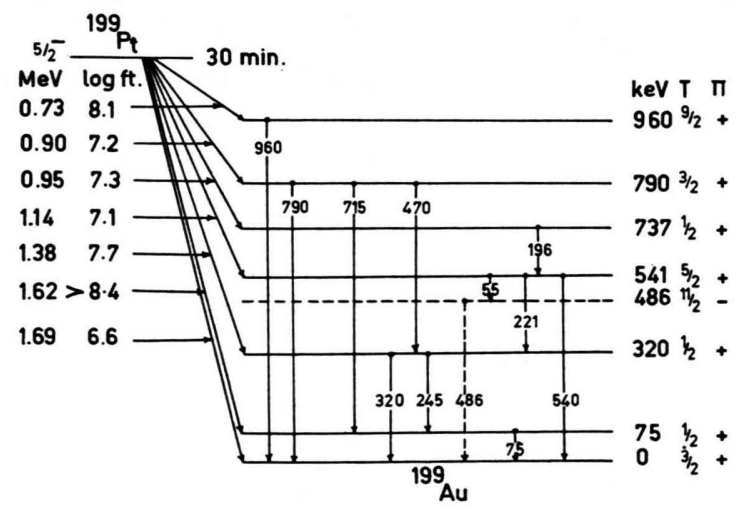

Fig. 4. Energy levels of ${ }^{199} \mathrm{Au}$ as populated in the decay of 30 min. ${ }^{199} \mathrm{Pt}$.

9 L. A. Surv and I. M. BAND, In Alpha-beta, and gamma-ray spectroscopy (Ed. K. Siegrahn), North Holland Publ. Co., Amsterdam 1965, Appendix 5. tions, where the $\mathrm{K}$ - and L-subshell ratios agreed with pure $\mathrm{E} 2$ transitions as shown in Table 2. The absolute K-conversion coefficients of the other transitions obtained from our measurements are used to determine the multipolarities by comparison with the theoretical values ${ }^{9}$. The results show that the 541 and $790 \mathrm{keV}$ transitions have magnetic dipole character while the $960 \mathrm{keV}$ transition has a magnetic octupole character, see Table 2.

The energy levels of ${ }^{199} \mathrm{Au}$ are given in Fig. 4. These were established earlier ${ }^{1,6}$ except the level shown by a dashed line. The ground state of ${ }^{199} \mathrm{Au}$ is shown to be $3 / 2+$ as measured by the atomic beam method ${ }^{10}$. The earlier measurements regarding the energy levels of ${ }^{199} \mathrm{Pt}$ have indicated that the spin and parity of its ground state is $5 / 2-$. From the systematics of odd-mass $\mathrm{Au}$ isotopes and the high $\log f t$ value $(>8.4)$ of the $\beta$ transition to the first excited state ${ }^{1}$ of ${ }^{199} \mathrm{Au}$, it seems probable that the spin and parity of this state is $1 / 2+$. The $320 \mathrm{keV}$ level may be assigned as $1 / 2+$ or $3 / 2+$. The spin and parity assignments of the higher levels in ${ }^{199} \mathrm{Au}$ have been made on the basis of these known assigned levels, the $\log f t$ values and the multipolarities obtained in the present study.

The $541 \mathrm{keV}$ level, $5 / 2+:$ The $541 \mathrm{keV}$ excited state is populated by the $\beta$ decay of ${ }^{199} \mathrm{Pt}$, and the $\log f t$ value corresponds to a first-forbidden character of this transition ${ }^{\mathbf{1}}$. It is also populated by the $196 \mathrm{keV}$ transition from the $737 \mathrm{keV}$ excited state. Evidence for spin $5 / 2+, 7 / 2+$ or $3 / 2+$ assignments can be derived from the log $f t$ of the $\beta$ transition to the $541 \mathrm{keV}$ level. The energy of the $541 \mathrm{keV}$ excited state in ${ }^{199} \mathrm{Au}$ is proposed due to the deexciting $\gamma$ ray of energy $(541.20 \pm 0.27) \mathrm{keV}$ found in our measurements instead of the $544 \mathrm{keV}$ transition suggested by Prasad et al. ${ }^{6}$. The magnetic dipole character of the $541 \mathrm{keV} \gamma$ ray excludes the the $7 / 2+$ assignment to this level. There is a $\gamma$ ray with energy $(221.21 \pm 0.11) \mathrm{keV}$ as has been observed in our internal and external conversion spectra. Such a transition is compatible with the weak $220 \mathrm{keV}$ transition of $30 \mathrm{~min}$ half-life reported by Prasad et al. ${ }^{6}$, but they could not replace it in the level scheme of ${ }^{199} \mathrm{Au}$, because it was difficult to observe in coincidence measurements. However, its

10 R. L. Christensen et al., Phys. Rev. 101, 1389 [1965]. 
energy fits very well with the location between the 541 and $320 \mathrm{keV}$ excited levels. The K/L- and Lsubshell ratios proved that it is a pure $\mathrm{E} 2$ transition. Since the $320 \mathrm{keV}$ level is assigned $1 / 2+$ or $3 / 2+$, therefore $5 / 2+$ is the most probable value for the $541 \mathrm{keV}$ excited level in ${ }^{199} \mathrm{Au}$. Consequently, the $320 \mathrm{keV}$ level might have $1 / 2+$ assignment. This is also consistent with the recent assignment by PRASAD et al. ${ }^{6}$.

The $737 \mathrm{keV}$ level, $1 / 2+$ : The $737 \mathrm{keV}$ excited state is shown to decay to the $54 \mathrm{l} \mathrm{keV}(5 / 2+)$ level via the $(195.6 \pm 0.1) \mathrm{keV}$ transitions; the transition has been observed before ${ }^{2}$. However, Prasad et al. ${ }^{6}$ did not mention it in their recent work. They observed instead a $\gamma$ ray of energy $192 \mathrm{keV}$. The appearance of this new energy needs more confirmation since there is a $192 \mathrm{keV} \gamma$ transition in ${ }^{197} \mathrm{Au}$ following the decay of ${ }^{197} \mathrm{Pt}$. The E 2 character of the $195.6 \mathrm{keV}$ transition infers a spin and parity of $1 / 2+$ for the $737 \mathrm{keV}$ level in ${ }^{199} \mathrm{Au}$.

The $790 \mathrm{keV}$ level, $3 / 2+$ : This level is mainly deexcited to the ground state by direct transition and by the cascading $715-75 \mathrm{keV}$ and $470-320 \mathrm{keV}$ transitions. From the K-conversion coefficient of the ground state $790 \mathrm{keV}$ transition, it was confirmed that it has a magnetic dipole character. Consequently the spin and parity $1 / 2+, 3 / 2+$ or $5 / 2+$ assignments are possible for the $790 \mathrm{keV}$ level. The $\log f t$ value (7.2) of the $\beta$ branch feeding this level is consistent with a first forbidden transition. Accordingly, $1 / 2+$ could be excluded. Directional correlation results ${ }^{6}$ favoured a $3 / 2+$ assignment to the $790 \mathrm{keV}$ level.

The $960 \mathrm{keV}$ level, 9/2 + : The measured K-internal conversion line intensity and $\gamma$-ray intensity of the $960 \mathrm{keV}$ transition gave the conversion coeffi- cient $\alpha_{K}=0.062 \pm 0.008$, which agrees with the theoretical coefficient for M3. From the multipolarity of the $960 \mathrm{keV}$ transition determined in our investigation and the properties of the ${ }^{199} \mathrm{Au}$ ground state, spin and parity $9 / 2+$ are estimated for the $960 \mathrm{keV}$ level. The $960 \mathrm{keV}$ excited state is fed by a $\beta$ branch with $\log f t=8.1$, wich could be considered as a unique, first-forbidden $\beta$ transition with $\Delta J=$ \pm 2 , and the parity of the successive level should be changed. Perhaps these data confirm the $9 / 2+$ assignment given to the $960 \mathrm{keV}$ excited level in ${ }^{199} \mathrm{Au}$.

The study of the nature of the various transitions shows that the level structure in ${ }^{199} \mathrm{Au}$ is quite complex. There is still a lack of experimental data especially for the determination of the isomeric state in ${ }^{199} \mathrm{Au}$. First Joshr et al. ${ }^{2}$ determined the isomeric state at $475 \mathrm{keV}$, and recently, after B $\mathrm{LACK}^{7}$ has reported a highly-converted $55 \mathrm{keV}$ transition found in the decay of ${ }^{199} \mathrm{Pt}$, Prasad et al. ${ }^{6}$ indicated the isomeric state at $549 \mathrm{keV}$, Secondly, the $187 \mathrm{keV} \gamma$-ray determined recently by PRASAD et al. ${ }^{6}$ has not been observed in $\gamma-\gamma$ coincidence measurement. In addition the $\gamma$ ray of energy $53 \mathrm{keV}$ mentioned in the cascade $187-53-497 \mathrm{keV}$, which was proposed in their decay scheme ${ }^{6}$, has not been observed in their investigations.

Due to the fact that the $55 \mathrm{keV}$ transition belongs ${ }^{7}$ to the decay of ${ }^{199} \mathrm{Pt}$, we suggest that an isomeric state might exist at $486 \mathrm{keV}$, see Fig. 4 .

In conclusion it can be said that some levels of the proposed decay scheme of ${ }^{199} \mathrm{Pt}$ were determined ${ }^{1,6}$ using less accurate data. Hence more accurate energies, coincidence and angular correlation measurements would be very valuable to complete this level scheme. 